\title{
Synthesis of 2-Dithioacetalmethylene-3-oxobutyronitrile from 2-Dithioacetalmethylene-3-oxobutyramide
}

\author{
Yu-Zi Jin, Qun Liu, ${ }^{+\bullet}$ Jing Dong Zhang, and Youn-Sik Lee ${ }^{\ddagger . *}$ \\ Deparment of Chemistry, College of Science, Ianbian Cniversitv, Yanji 133-002, P.R. China \\ ${ }^{\dagger}$ Department of Chemistry, Northeast Normal Chiversitv, Changchun 130-024, P.R. China \\ ${ }^{\ddagger}$ Diwision of Emironmental and Chemical Engineering, Nanomaterials Research Center, Chonbuk National Lniversity, \\ Chonit 561-756, Korea. "E-mail: vosklearachonbukac.kr \\ Received November 13, 2006
}

Key Words : 2-Dithioacetalmethỵlene-3-oxobutyramide. 2-Dithioacetalmethỵlene-3-oxobuty ronitrile, $\alpha$-Cyano$\alpha$-cinnamoylketene dithioacetal

Nitriles are important building blocks in the construction of amines, amides. ketones. carboxylic acids. and esters. For example. 1,2-diarylimidazoles as the potent anti-inflammatory agent, ${ }^{1}$ thiazole analogues as the inhibitor of superoxide. ${ }^{2}$ benzanidines with activity of fibrinogen antagonist. ${ }^{3}$ $(R)$-anides and $(S)$-acids as the enzyme. ${ }^{+}$and glutarimides with pharmacological effects ${ }^{5}$ were all prepared from nitriles.

Occasionally, nitriles have been synthesized from aldehyde. For example, catalytic dehydration of aldoximes provided various types of cyano compounds. ${ }^{6.5} \mathrm{O}$-Substituted aldoximes, formed by the reaction between aldehydes and 2.4-dinitropheny lhydroxylamine. underwent 1.2-elimination in the presence of a base to give the corresponding nitriles. ${ }^{11}$ Bose and Narsaiah synthesized nitriles in one-pot from aldehydes in a solid state, using peroxymonosulfate on alumina. ${ }^{11-15}$ Kaneda et al. reported one-pot synthesis of $\alpha$ alkylated nitriles with carbonyl compounds, via aldol reaction-lydrogenation, using hydrotalcite-supported palladium nanoparticles as multifunctional catayst. ${ }^{16}$ However, most of these methods suffer from the limited availability of starting materials. low reaction yields, drastic reaction conditions. or tedious work-up procedures.

Herein. we report a synthetic route to 2-dithioacetalmethylene-3-oxobutyronitriles from 2-dithioacetalmethylene3-oxobutyramides. as shown in Scheme 1. The resulting nitriles contain an unstable $\alpha$-acetoketene dithioacetal moiety, belonging to a class of $a$-oxoletene dithioacetals which are well-known for organic syntheses. ${ }^{17.19}$ and thus can be employed as new intermediates for the syntheses of more complicated organic compounds. In fact. it was reported that l-acetyl cyclopropane carbosamide was converted to 1- acetyl cyclopropane carbonitrile, using methanesulfonyl clloride in the presence of pyridine. ${ }^{30}$ In comparison to acetyl chloride, however, methanesulfonyl chloride is more expensive and corrosive.

\section{Results and Discussion}

As shown in Scheme 1, we successfully converted 2dithioacetalmethylene-3-oxobutyramide (1) to 2-dithioacetalmethylene-3-oxobutyronitrile (2) using acetyl chloride. Briefly, 1 was refluxed with acetyl chloride (0.5 equiv) in toluene for about 7-10 h to give 2-dithioacetalmetlyylene-3oxobutyronitrile 2 in $85-90 \%$ yields (Table 1) along with a small amount of byproduct resulted from the removal of the alkylthio groups. 2a, containing a 5-membered ring of dithioacetal moiety $\left(\mathrm{R}=\mathrm{CH}_{2} \mathrm{CH}_{2}\right)$, was obtained as a solid, after chromatographic separation of the reaction residue. The FT-IR spectrum of 2a showed a characteristic absorption peak of cyano group at $2200 \mathrm{~cm}^{-1}$. The ${ }^{13} \mathrm{C}$-NMR spectrum showed a carbon resonance peak of cyano group at $117.8 \mathrm{ppm}$. The combined two spectral data confirmed the expected chemical structure of the isolated $2 \mathbf{a}$.

After the synthesis of compound 2a. we attempted to synthesize different analogs, containing a 6- or 7-membered ring of ketene dithioacetal moiety. $\mathbf{2 b}$ and $\mathbf{2} \mathbf{c}$ were synthesized under similar conditions in $90 \%$ and $85 \%$ yields, respectively. Finally. we also attempted to synthesize other analogs. containing acyclic ketene dithioacetal moieties. $2 \mathrm{~d}$ $\left(\mathrm{R}=\mathrm{CH}_{3}\right)$ and $2 \mathbf{e}\left(\mathrm{R}=\mathrm{C}_{6} \mathrm{H}_{5} \mathrm{CH}_{2}\right)$ were successfully synthesized in $86 \%$ and $89 \%$ yields. respectively. This result indicates that the chemical reaction of $\mathbf{1}$ with acetyl chloride

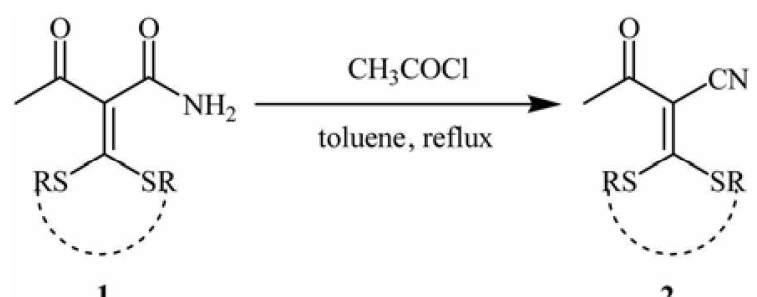

Scheme 1<smiles>CC(=O)C(C#N)=C1SCCS1</smiles>

$2 \mathrm{a}$

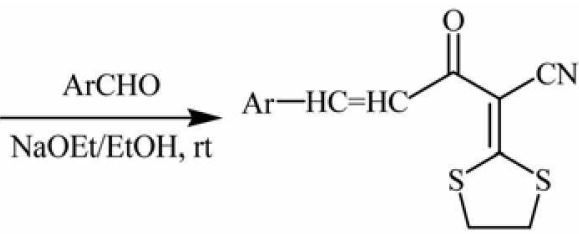

3

Scheme 2 
Table 1. Sunthesis of 2-dithioacetalmethvlene-3-oxobutyronitriles (2a-e) and $\alpha$-cyano- $\alpha$-cimnamoyl dithoacetals (3a-c)

\begin{tabular}{cccccc}
\hline Intermediate & $\mathrm{R}$ & $\mathrm{Ar}$ & Product & $\begin{array}{c}\text { Time } \\
(\mathbf{h})\end{array}$ & $\begin{array}{c}\text { Yield } \\
(\%)\end{array}$ \\
\hline 1a & $\left(\mathrm{CH}_{2}\right)_{2}$ & - & $2 \mathrm{a}$ & 8 & 89 \\
1b & $\left(\mathrm{CH}_{2}\right)_{2}$ & - & $2 \mathrm{~b}$ & 10 & 90 \\
1c & $\left(\mathrm{CH}_{2}\right)_{4}$ & - & $2 \mathrm{c}$ & 7 & 85 \\
1d & $\mathrm{CH}_{3}$ & - & $2 \mathrm{~d}$ & 7 & 86 \\
1e & $\mathrm{C}_{6} \mathrm{H}_{5} \mathrm{CH}_{2}$ & - & $2 \mathrm{e}$ & 8 & 89 \\
2a & $\left(\mathrm{CH}_{2}\right)_{2}$ & 2-Pyridiny] & $3 \mathrm{a}$ & 1 & 82 \\
2a & $\left(\mathrm{CH}_{2}\right)_{2}$ & $4-\mathrm{CH}_{3} \mathrm{OC}_{6} \mathrm{H}_{4}$ & $3 \mathrm{~b}$ & 1 & 89 \\
2a & $\left(\mathrm{CH}_{2}\right)_{2}$ & $4-\mathrm{NO}_{2} \mathrm{OC}_{6} \mathrm{H}_{4}$ & $3 \mathrm{c}$ & 0.5 & 77 \\
\hline
\end{tabular}

"Based on the isolated product

is not much affected by the structure (ring size. cyclic or acyclic) of ketene dithioacetal moiety.

In order to investigate any possibility of further functionalization of 2. 2a was reacted with various benzaldelydes (ArCHO), such as picolinaldehy'de 4-methoxy benzaldehyde and 4-nitrobenzaldehyde. as shown in Scheme 2. Briefly 2a (1.0 equiv) was reacted with $\mathrm{ArCHO}$ (1.1 equiv) in the presence of $\mathrm{NaOEt}(0.5$ equiv) in ethanol at room temperature (Table 1). The isolation yields after column chromatographyic separation were moderate to high $(77-89 \%)$. This result indicates that the reactions are not significantly affected either by the electron-releasing or electron-withdrawing effect of substituents in the ArCHO.

The $\alpha$-cyano- $\alpha$-cinnamoyl dithioacetals 3a-c have unique structural characteristics. They have five active carbons that are all conjugated $(\mathrm{ArCH}=\mathbf{C H}-\mathrm{CO}-\mathrm{C}(\mathrm{CN})=\mathbf{C S}$ ) (double Michael sy'stem). and thus can undergo the Michael addition in two different ways. In addition, the cyano group in these compounds can be converted to an amino group via hydrogenation. The resulting intermediates. containing the amino group. can then undergo intramolecular cycloaddition to form the corresponding aza-cyclic compounds.

For the preparation of nitriles from primary amides. Lew is acids have been often employed under a vigorous reaction condition. ${ }^{31}$ In a recent examiple. Nakajima and co-workers

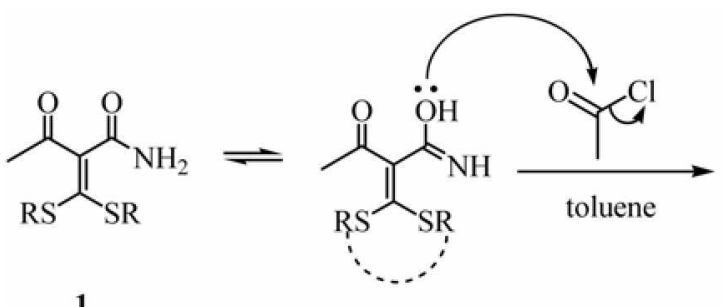

1

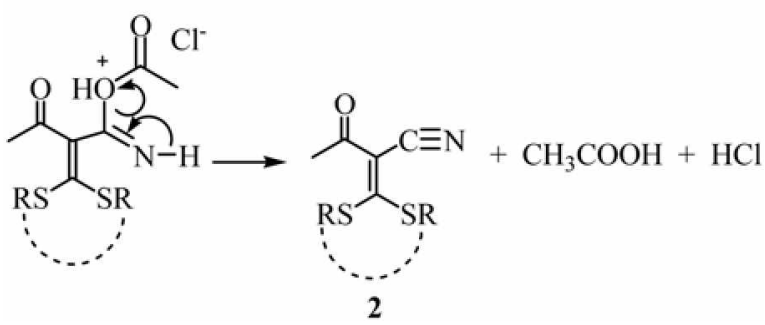

sy'nthesized nitriles by reacting primary amides with $(\mathrm{COCl})_{2}=$ activated dimethyl sulfoxide. in the presence of triethylamine at $-78^{\circ} \mathrm{C}$. We proposed a mechanism for the conversion of the amide group to cyano group with acetyl chloride as shown in Scheme 3. Acetyl chloride reacts with amino-carbonyl oxygen of 2-dithioacetalmethylene-3-oxobutyramide. to form the corresponding adduct salt. Subsequently, the salt undergoes elimination to give the corresponding nitrile product along with acetic acid and hydrochloride.

In conclusion. the reaction of 2-dithioacetalmethylene-3oxobutyramides with acetyl clloride is an effective method for the synthesis of 2-dithioacetalmethylene-3-oxobutyronitriles. The nitriles were further converted to $\alpha$-cyano- $\alpha$ cinnamoyl dithoacetals via crossed aldol reaction with benzaldehydes. The synthesized $\alpha$-cyano- $\alpha$-cinnamoyl dithoacetals can be employed as new precursors for synthesis of more functionalized compounds

\section{Experimental Section}

All reagents were purchased from the Aldrich Chemical Co.. and used without further purification unless otherwise noted. A solution of $0.1 \mathrm{~N} \mathrm{NaOEt}$ in $\mathrm{EtOH}$ was prepared inmediately prior to use. The products were purified by column chromatography over silica gel purchased from the Qingdao Ocean Chemical Co. 1 was prepared according to a known method. ${ }^{17.19}$ Melting points were not corrected. ${ }^{1} \mathrm{H}$ NMR and ${ }^{13} \mathrm{C}$ NMR spectra were recorded on a Bruker Avance-600, $400 \mathrm{NMR}$ spectrometer. using $\mathrm{CDCl}_{3}$ and TMS as solvent and intemal standard. respectively. IR spectra were recorded on a Magna-560 FT-IR spectrophotometer in the range of $400-4000 \mathrm{~cm}^{-1}$. using $\mathrm{KBr}$ pellets. Mass spectra (MS) were recorded on a Gas Chromatography/Mass Spectrometer (HP6890/5973). Elemental analyses were measured on a Vario EL analyzer.

General Procedure for the Synthesis of 2. A $100-\mathrm{mL}$ three-necked flask was equipped with a reflux condenser. a sealed stirrer unit and a thermometer. Toluene $(98 \%, 20$ $\mathrm{mL}$ ). acetyl chloride $(785 \mathrm{mg} .10 \mathrm{mmol})$ and 2-dithioacetalmetly lene-3-oxobutyramide $1(0.4 \mathrm{~g} .2 .0 \mathrm{mmol})$ were transferred into the flask. The solution was refluxed for $7-10 \mathrm{~h}$. The solvent was removed under reduced pressure to obtain crude product. which was then column cluromatographed over silica gel to give compound 2 (hexane-EtOAc. 85/15).

2-(1.3-Dithiolan-2-ylidene)-3-oxobutyronitrile (2a): yellow crystal: mp 145-147 ${ }^{\circ} \mathrm{C}$ (recrystallization from hexanemethane. $5 / 3$ ): $\mathrm{R}_{\mathrm{f}}=0.3$ (hexane-EtOAc. $3 / 2$ ): IR $(\mathrm{KBr}$ ): 2200. 1662, 1441. $1274 \mathrm{~cm}^{-1} ;{ }^{1} \mathrm{H}$ NMR $\left(600 \mathrm{MHz}, \mathrm{CDCl}_{3}\right)$ : $\delta 2.43\left(\mathrm{~s}, 3 \mathrm{H} . \mathrm{CH}_{3}\right) .3 .54\left(\mathrm{~m}, 2 \mathrm{H} . \mathrm{SCH}_{2}\right) .3 .59$ (m. $2 \mathrm{H}$. $\left.\mathrm{SCH}_{2}\right):{ }^{13} \mathrm{C}$ NMR $\left(100 \mathrm{MHz} . \mathrm{CDCl}_{3}\right): \delta 27.9,37.3,40.5$, 97.8. 117.8, 183.9, 189.9; MS: $\mathrm{m} / \mathrm{z}(\%) .185(\mathrm{M}, 100 \%)$; Anal. calcd for $\mathrm{C}_{7} \mathrm{H}_{7} \mathrm{NOS}_{2}: \mathrm{C}, 45.38 ; \mathrm{H}, 3.81 ; \mathrm{N}, 7.56$. Found: C, 48.07: H, 4.54: N. 7.04

2-(1.3-Dithian-2-ylidene)-3-oxobutyronitrile (2b): $\mathrm{mp}$ $110-112^{\circ} \mathrm{C}$ (recrystallization from hexane-methane. $5 / 3$ ): $\mathrm{R}_{\mathrm{f}}$ $=0.5$ (hexane-EtOAc. 1/1): IR (KBr): 2197, 1647, 1416. 
$1263 \mathrm{~cm}^{-1} ;{ }^{1} \mathrm{H}$ NMR $\left(400 \mathrm{MHz} . \mathrm{CDCl}_{3}\right): \delta 2.30-2.37(\mathrm{~m}$. $2 \mathrm{H} . \mathrm{SCCH}_{2} \mathrm{CS}$ ). 2.42 (s. $3 \mathrm{H} . \mathrm{CH}_{3}$ ). 2.99 (t. $2 \mathrm{H} . J=8.0 \mathrm{~Hz}$. $\left.\mathrm{SCH}_{2}\right), 3.13\left(\mathrm{t}, 2 \mathrm{H} . J=8.0 \mathrm{~Hz}, \mathrm{SCH}_{2}\right),{ }^{13} \mathrm{C}$ NMR $(100 \mathrm{MHz}$. $\left.\mathrm{CDCl}_{3}\right): \delta 28.9,29.7(2 \mathrm{C}), 29.8 .105 .1,117.3 .181 .1,190.4$ : MS: $\mathrm{m} / \mathrm{z}(\%) .199(\mathrm{M} .100 \%)$ : Anal. calcd for $\mathrm{C}_{8} \mathrm{H}_{9} \mathrm{NOS}_{2} \mathrm{C}$. 48.21: H. 4.55: N. 7.03. Found: C. 47.07: H. 3.34: N. 7.04

2-(1.3-Dithiepan-2-ylidene)-3-oxobuty ronitrile (2c): $\mathrm{mp}$ $87-89^{\circ} \mathrm{C}$ (recrystallization from hexane-methane, $5 / 3$ ): $R_{\mathrm{f}}=$ 0.6 (hexane-EtOAc. 3/2): IR ( $\mathrm{KBr}$ ): 2237, 1607, 1416, 1253 $\mathrm{cm}^{-1} ;{ }^{1} \mathrm{H}$ NMR $\left(300 \mathrm{MHz} . \mathrm{CDCl}_{3}\right): \delta 2.28-2.35$ (m. $4 \mathrm{H}$. $\mathrm{SCCH}_{2} \mathrm{CCH}_{2} \mathrm{CS}$ ), 2.38 (m. $\left.2 \mathrm{H} . \mathrm{CH}_{2}\right), 2.40$ (s, $3 \mathrm{H}_{3} \mathrm{CH}_{3}$ ), 3.10 (t. $2 \mathrm{H}, J=7.8 \mathrm{~Hz} . \mathrm{SCH}_{2}$ ) 3.15 (t. $2 \mathrm{H}, J=7.8 \mathrm{~Hz}$. $\left.\mathrm{SCH}_{2}\right):{ }^{13} \mathrm{C}$ NMR $\left(100 \mathrm{MHz} . \mathrm{CDCl}_{3}\right): \delta 28.9,29.8 .29 .8$. 29.9. 30.1. 103.1.117.5. 180.6. 188.4: MS: $\mathrm{m} / \mathrm{z}(\%) .476(\mathrm{M}$. $90 \%$ ): Anal calcd for $\mathrm{C}_{9} \mathrm{H}_{11} \mathrm{NOS}_{2}: \mathrm{C}, 50.67 ; \mathrm{H}, 5.20 ; \mathrm{N}$. 6.57. Found: C. 50.53: H. 5.20: N. 6.53 .

2-(Bis(methylthio)methylene)-3-oxobuty ronitrile (2d): $\mathrm{mp}$ $56-58^{\circ} \mathrm{C}$ (recrystallization from hexane-methane, $5 / 3$ ): $\mathrm{R}_{\mathrm{f}}=$ 0.3 (hexane-EtOAc. 6/1): IR (KBr): 2201. 1666. 1419. 1250 $\mathrm{cm}^{-1},{ }^{\mathrm{H}} \mathrm{H} \mathrm{NMR}\left(400 \mathrm{MHz}, \mathrm{CDCl}_{3}\right): \delta 2.47\left(\right.$ s. $\left.3 \mathrm{H}, \mathrm{CH}_{3}\right), 2.59$ (s. $\left.3 \mathrm{H}, \mathrm{CH}_{3}\right), 2.78\left(\mathrm{~s}, 3 \mathrm{H} . \mathrm{CH}_{3}\right) ;{ }^{13} \mathrm{C}$ NMR $(100 \mathrm{MHz}$. $\left.\mathrm{CDCl}_{3}\right): \delta 28.9 .29 .8 .29 .9,103.1,117.5 .180 .6$ 188.4: MS: $\mathrm{m} / \mathrm{z}(\%) .187$ (M. $25 \%$ ): Anal calcd for $\mathrm{C}_{7} \mathrm{H}_{9} \mathrm{NOS}_{2}$ : C. 44.89: H. 4.84: N. 7.48. Found: C. 43.98: H. 4.84: N. 7.50.

2-(Bis(benzylthio)methylene)-3-oxobutyronitrile (2e): $\mathrm{mp}$ $58-60{ }^{\circ} \mathrm{C}$ (recrystallization from hexane-methane, $5 / 3$ ): $\mathrm{R}_{\mathrm{f}}=$ 0.3 (hexane-EtOAc. 3/2): IR (KBr): 2201, 1668, 1439, 1249 $\mathrm{cm}^{-1},{ }^{1} \mathrm{H} \mathrm{NMR}\left(400 \mathrm{MHz}, \mathrm{CDCl}_{3}\right): \delta 2.44$ (s. $\left.3 \mathrm{H}, \mathrm{CH}_{3}\right) .4 .20$ (s. $\left.2 \mathrm{H} \mathrm{SCH}_{2}\right), 4.39$ (s. $\left.2 \mathrm{H}_{1} \mathrm{SCH}_{2}\right), 7.20-7.36$ (m. $10 \mathrm{H}, \mathrm{ArH}$ ): ${ }^{13} \mathrm{C} \mathrm{NMR}\left(100 \mathrm{MHz}, \mathrm{CDCl}_{3}\right): \delta 29.2 .40 .8(2 \mathrm{C}) .42 .9(2 \mathrm{C})$. 109.5. 118.3. 128.0-134.9 (10 C), 176.2. 191.1: MS: $\mathrm{m} / \mathrm{z}$ (\%). 339 (M. 80\%): Anal. calcd for $\mathrm{C}_{19} \mathrm{H}_{17} \mathrm{NOS}_{2}$ : C. 67.22 ; H. 5.05: N. 4.13. Found: C. 67.81: H. 4.85: N 4.24 .

General Procedure for the Synthesis of 3 from 2a. To a stirred suspension of 2-(1.3-dithiolan-2-y lidene)-3-oxobuty ronitrile (2a) $(2.0 \mathrm{~mm}$ ) $)$ in ethanol $(15 \mathrm{~mL})$. various arylaldehydes (2.2 mmol) and a fresh solution of $0.1 \mathrm{~N} \mathrm{NaOEt}$ in $\mathrm{E} \mathrm{OH}(10 \mathrm{~mL})$ were added. The mixture was stirred at room temperature for about $1 \mathrm{~h}$. The solvent was removed under reduced pressure. when the starting material. 2a disappeared (checked by TLC). The residue was washed with water $(3 \times$ $15 \mathrm{~mL}$ ) and then dried. The crude product was column chromatographed on silica gel (hexane-EtOAc. 90/10), and recrystallized from hexane-EtOAc (3/2) to give 3 .

2-(1.3-Dithiolan-2-ylidene)-3-oxo-5-(py ridin-2-yl)pent-4enenitrile (3a): $\mathrm{mp} 168-170^{\circ} \mathrm{C}$ (recrystallization from hexanemethane. 2/1): $\mathrm{R}_{\mathrm{f}}=0.3$ (hexane-EtOAc. 1/2): IR $(\mathrm{KBr})$ : 2202. 1648, 1430. 1330. $122 \mathrm{I} \mathrm{cm}^{-1}:{ }^{1} \mathrm{H}$ NMR $(400 \mathrm{MHz}$. $\left.\mathrm{CDCl}_{3}\right): \delta 3.56-3.65\left(\mathrm{~m}, 4 \mathrm{H} . \mathrm{SCH}_{2}\right), 7.28(\mathrm{~m}, \mathrm{lH} . \mathrm{ArH})$. 7.50 (d, IH. $J=8.0$. ArH). $7.70(\mathrm{~m}, \mathrm{lH} . \mathrm{ArH}), 7.77$ (d, $1 \mathrm{H} . J$ $=12.0 \mathrm{~Hz},=\mathrm{CH}) .7 .85(\mathrm{~d} .1 \mathrm{H} . J=12.0 \mathrm{~Hz}=\mathrm{CH}) .7 .67(\mathrm{~d}$. lH. $J=4.0 \mathrm{~Hz}, \mathrm{ArH}): \mathrm{MS}: \mathrm{m} / \mathrm{z}(\%) .274$ (M. 15\%): Anal. calcd for $\mathrm{C}_{13} \mathrm{H}_{10} \mathrm{~N}_{2} \mathrm{OS}_{2}: \mathrm{C}, 56.91 ; \mathrm{H} .3 .67 ;$ N. 10.21 . Found:
C. $56.32: H, 3.57 ;$ N. 10.50 .

2-(1,3-Dithiolan-2-ylidene)-5-(4-methoxyphenyl)-3-oxopent-4-enenitrile (3b): $\mathrm{mp} 205-20{ }^{\circ} \mathrm{C}$ (recrystallization from hexane-methane. $2 / 1$ ): $R_{f}=0.3$ (hexane-EtOAc. $1 / 2$ ); IR (KBr): 2197, 1645. 1434. 1510.1256 $\mathrm{cm}^{-1} .{ }^{1} \mathrm{H}$ NMR $(600$ $\left.\mathrm{MHz}, \mathrm{CDCl}_{3}\right): \delta 3.54-3.62\left(\mathrm{~m} .4 \mathrm{H}, \mathrm{SCH}_{2}\right) .3 .86(\mathrm{~s}, 3 \mathrm{H}$. $\left.\mathrm{OCH}_{3}\right) .6 .91$ (d. $\left.1 \mathrm{H} . J=12.0 \mathrm{~Hz} .=\mathrm{CH}\right), 7.25(\mathrm{~d} .1 \mathrm{H}, J=6.0$, $\operatorname{ArH}) .7 .58$ (d. $1 \mathrm{H} . J=6.0 . \mathrm{ArH}) .7 .76(\mathrm{~d}, 1 \mathrm{H} . J=12.0 \mathrm{~Hz}$. $=\mathrm{CH}):$ MS: $\mathrm{n} / \mathrm{z}(\%) .291$ (M, 18\%): Anal. calcd for $\mathrm{C}_{15} \mathrm{H}_{13} \mathrm{NO}_{2} \mathrm{~S}_{2}: \mathrm{C}, 59.38$ : H. 4.32; N, 4.62. Found: C, 57.61; H. $4.25:$ N. 4.50 .

2-(1.3-Dithiolan-2-ylidene)-5-(4-nitropheny1)-3-oxo-pent4-enenitrile (3c): $\mathrm{mp} 256-258{ }^{\circ} \mathrm{C}$ (recrystallization from hexane-methane. 2/1): $R_{f}=0.3$ (hexane-EtOAc, $1 / 3$ ); IR (KBr): 2207. 1635. 1335, 1210,840 $\mathrm{cm}^{-1}:{ }^{1} \mathrm{H}$ NMR $(400$ $\left.\mathrm{MHz}, \mathrm{CDCl}_{3}\right): \delta 3.72(\mathrm{~m}, 4 \mathrm{H} . \mathrm{SCH}), 7.41(\mathrm{~d}, \mathrm{lH}, J=15.6$ $\mathrm{Hz}=\mathrm{CH}$ ). 7.43 (d. $1 \mathrm{H} . J=15.6 \mathrm{~Hz}=\mathrm{CH}$ ). 7.97 (d. $1 \mathrm{H} . J=$ $8.7 \mathrm{~Hz}, \mathrm{ArH}$ ). 8.25 (d. $1 \mathrm{H}, J=8.7 \mathrm{~Hz}$. ArH): MS: $\mathrm{m} / \mathrm{z}(\%)$. 306 (M. 21\%): Anal calcd for $\mathrm{C}_{14} \mathrm{H}_{10} \mathrm{~N}_{2} \mathrm{O}_{3} \mathrm{~S}_{2}: \mathrm{C}, 52.82: \mathrm{H}$. 3.17: N. 8.08. Found: C. 52.80: H. 3.15: N. 8.44

Acknowledgements. Financial support of this research was provided by the NNSFC (29862004) in P. R. China and KOSEF (Grant No. R01-2004-000-10390-0) in Korea.

\section{References}

1. Fabiani. M. E. Dntg New's Prospect 1999. 12.207.

2. Chichiro. M.: Nagamoto. H.: Takemura. I.: Kitano. K.: Komatsu. H.: Sekiguchi. K.: Tabusa. F.: Mori. T.: Tominnaga. M.: Yabuuchi. Y. J.Med Chent 1995. 38. 353.

3. Judkins. B. D.: Allen, D. G.; Cook, T. A.; Evans. B.; Sardharwala. T. E. Synth Commun. 1996, 26, 4351.

4. Zhong-Liu. W.: Zu-Y. L. Tetrahedron: Astmmety 2001. 12. 3305 .

5. Takaya. H.: Yoshida. K.: Isozahi. K.: Terai. H.: Murahashi. S.-I. Angew. Chem., Hnt Ed. 2003. 22.3302 .

6. Movassagh. B.: Shokri. S. Symh. Commm. 2005, $35,887$.

7. Yang. S. H.: Chang, S. Org. Lett. 2001. 3. 4209.

8. Thomas. H. G.; Grevn. H. D. Smblesis 1990. 129.

9. Desai. D. G.: Swami. S. S.: Mahale. G. D. Smm Commm 2000. 30.1623 .

10. Miller. M. T.: Loudon. G. M. J. Org. Chent 1975. 40. 126.

11. Das. B.; Ramesh, C.: Madhusudhan, P. Synlett 2000. 1599.

12. Wang, E.-C.: Lin, G.-J. Tetrahedron Lett $1998.39,4047$.

13. Lai. G.: Bhamare. N. K.: Anderson. W. K. Smlett 2001. 230.

14. Bose. D. S.: Narsaiah. A. V. Tetrahedron Lett. 1998. 39.6533

15. Srinivas. K. V. N. S.: Reddy. E. B.: Das. B. Swlen 2002. 625

16. Motokura. K.: Fujita. N.: Mori. K.: Mizugaki. T.: Ebitani. K.: Kaneda. K. Tetrahedron Lett. 2005, $16,5507$.

17. Dieter. R. K. Terahedron 1986, +2, 3029 .

18. Junjappa. H.: Ila. H.: Asokan. C. V. Tetrohedhon 1990. 46.5423.

19. Mahata. P. K.: Syam Kumar. U. K.: Sriram. V: Ila. H.: Tunjappa. H. Tetrahedron 2003. 59. 2631

20. Makoto. M.: Yuichiro T.: Naoki. O.: Toshifumi. A. Japan Patent 20002069072 .

21. Lehnert, W. Tetrahedron Lett. 1971, 12, 1501

22. Nakajima, N.: Saito, M.: Utbukata, M. Tetrohedon 2002. 58.3561. 